\title{
Image sampling in static telepathology for frozen section diagnosis
}

\author{
Vincenzo Della Mea, Palmina Cataldi, Sebastiana Boi, Nicoletta Finato,
} Paolo Dalla Palma, Carlo A Beltrami

\begin{abstract}
Background-A frozen section diagnostic service is often not directly available in small rural or mountain hospitals. In these cases, it could be possible to provide frozen section diagnosis through telepathology systems. Telepathology is based on two main methods: static and dynamic. The former is less expensive, but involves the crucial problem of image sampling. Aims-To characterise the differences in image sampling for static telepathology when undertaken by pathologists with different experience.

Methods-As a test field, a previously studied telepathology method based on multimedia email was adopted. Using this method, three pathologists with different levels of experience sampled images from 155 routine frozen sections and sent them to a distant pathology institute, where diagnoses were made on digital images. After the telepathology diagnoses, the glass slides of both the frozen sections and the definitive sections were sent to the remote pathologists for review.

Results-Four of 155 transmissions were considered inadequate by the remote pathologist. In the remaining 151 cases, the telepathology diagnosis agreed with the gold standard in $146(96.7 \%)$. There was no significant divergence between the three pathologists in their sampling of the images. Each case comprised five images on average, acquired in four minutes. The overall time for transmission was about 19 minutes.
\end{abstract}

Conclusions-The results suggest that in routine frozen section diagnosis an inexperienced pathologist can sample images sufficiently well to permit remote diagnosis. However, as expected, the internet is too unreliable for such a time dependent task. An improvement in the system would involve integrated real time features, so that there could be interaction between the two pathologists.

(f Clin Pathol 1999;52:761-765)

Keywords: telepathology; internet; frozen sections

Institute of Pathology, S Chiara City

Hospital, Trento, Italy

S Boi

P Dalla Palma

Correspondence to:

Dr Della Mea

email:

dellamea@dimi.uniud.it

Accepted for publication 21 January 1999 Themendous growth in telematic niques and information systems has encouraged the development of new methods of medical practice. Thus in recent years digital images, multimedia, and electronic communication have become easily and cheaply available on the physician's desktop. Telemedicine, which is the practice of medicine at a distance by means of telematic tools, is one of the applications of the new technological advances.

The term telemedicine usually covers a variety of tasks, including telediagnosis, distant teaching and learning, remote image and data processing, distribution of case archives and so on. In the fields of clinical pathological practice, telediagnosis can be further divided into two main categories: expert consultation and intraoperative services. Both share the same basic activity - that is, the observation of specimens under the microscope to make a diagnosis and to provide highly specialised medical information to clinicians. However, there are of course some important differences.

Expert consultation is a common practice for dealing with those particularly rare or difficult cases that crop up during routine work: the glass slides, or sometimes the paraffin blocks, are sent to a recognised expert in the field by mail or courier, with evident problems of loss and damage. ${ }^{1}$ The expert consultation turnaround time - ranging from days to weeks - is not often a critical issue. The second opinion can be used to modify or to confirm the previous diagnosis, and is very important for the overall diagnostic quality of a department of pathology.

Intraoperative diagnosis using frozen sections, on the other hand, is in many cases necessary to establish a histopathological diagnosis, in order to guide the surgeon in performing the most appropriate surgical procedure. However, small hospitals (for example, those located in rural or mountain areas) often do not have an intraoperative diagnostic service, so they need some external support, such as a consulting service whereby a pathologist comes from another hospital during the surgery sessions, or by sending the tissue samples to an institute of pathology in an ambulance. Such methods sometimes cannot be used because of the geographic or climatic nature of the region. Moreover, a pathologist working alone in a peripheral hospital has no facilities for intradepartmental consultation - that is, consultation with other colleagues over difficult cases. This reduces the overall quality of the service. ${ }^{3} \mathrm{We}$ can thus distinguish two different needs: delivery of a complete frozen section service, and interactive support during frozen section diagnosis.

Both of these could be achieved by introduc- ing a telepathology link between the peripheral hospital and the department of pathology. The second can be further subdivided to include two options: sending a junior pathologist or a 
Table 1 Distribution of cases according to organ and referring pathologist

\begin{tabular}{lrrr}
\hline Organ & $(A)$ & $(B)$ & $(C)$ \\
\hline Appendix & - & 1 & - \\
Bladder & - & 1 & - \\
Brain & - & 3 & 2 \\
Breast & 19 & 16 & 13 \\
Bronchus & 2 & 2 & 1 \\
Diaphragm & - & 1 & - \\
Epiglottis & 1 & - & - \\
Liver & 2 & 2 & 8 \\
Lung & 2 & 1 & 2 \\
Lymph nodes & 10 & 9 & 11 \\
Mediastinum & 3 & - & - \\
Oesophagus & - & 1 & - \\
Omentum & - & 1 & - \\
Ovary & 2 & 9 & 3 \\
Pancreas & 1 & - & - \\
Peritoneum & - & 1 & - \\
Rectum & 1 & - & 6 \\
Stomach & 1 & 2 & 2 \\
Skin & 2 & - & - \\
Thyroid & 3 & 4 & 3 \\
Ureter & 1 & - & - \\
Total & $\mathbf{5 0}$ & $\mathbf{5 4}$ & $\mathbf{5 1}$
\end{tabular}

A, expert pathologist; B, junior pathologist; $C$, first year resident.

resident to the peripheral hospital with continuous online support of an expert pathologist located elsewhere, or supporting occasional online consultations with an expert pathologist.

Telepathology techniques suitable for carrying out diagnostic tasks are commonly subdivided into two categories: static telepathology (that is, based on still images), and dynamic telepathology (based on real time video).$^{4-7}$ Unsurprisingly, dynamic methods are more expensive than static ones in terms of both instrumentation and running costs.

The present report deals mainly with intraoperative static telepathology, though some of the topics considered are of general interest for any application of methods based on still images. This is because static telepathology, if proven to be adequate for pathology applications, can be truly cost-effective and thus affordable in diagnostic practice, mainly in relation to online consultations as described above.

Our aim in this study was to characterise the differences in image sampling for static telepathology when the sampling was done by pathologists with different levels of experience. After the gross sampling, image sampling is the most crucial step in static telepathology because it might guide the remote consultant to a wrong diagnosis. For this investigation, we adopted a transmission method based on the internet multimedia electronic mail. ${ }^{8}$

\section{Methods}

Two institutions were involved in this study: the department of pathology of the University of Udine, Italy, and the Institute of Pathology of S Chiara City Hospital, Trento, Italy (240

Table 2 Summary of results

\begin{tabular}{lclllll}
\hline $\begin{array}{l}\text { Case } \\
\text { selector }\end{array}$ & $\begin{array}{l}\text { No of } \\
\text { cases }\end{array}$ & Failed & Postponed & Agreement & No of images & $\begin{array}{l}\text { Acquisition } \\
\text { time per case }\end{array}$ \\
\hline A & 50 & 0 & 2 & $100 \%$ & $4(3$ to 6) & $4(2$ to 6) \\
B & 54 & 4 & 1 & $92.5 \%$ & $4.5(3$ to 5$)$ & $4(3$ to 4) \\
C & 51 & 1 & 1 & $97.9 \%$ & $5(4$ to 7) & $4(3$ to 6) \\
Total & $\mathbf{1 5 5}$ & $\mathbf{5}$ & $\mathbf{4}$ & $\mathbf{9 6 . 7 \%}$ & $\mathbf{5}$ (4 to 6) & $\mathbf{4}$ (3 to 6) \\
\hline
\end{tabular}

A, expert pathologist; B, junior pathologist; $\mathrm{C}$, first year resident. $\mathrm{km}$ from Udine). The former was the referring telepathology site, where pathologists sampled images from routine frozen sections and sent them by internet multimedia electronic mail; the latter was the consultant telepathology site, where two pathologists made diagnoses from images visualised on their computer monitors. After telepathological diagnosis, the results of which were not used for patient care, slides were sent to the consultant site, where definitive diagnoses were made.

At the referring site, three pathologists with different levels of expertise (an expert pathologist, a junior pathologist, and a first year resident in pathology) acted as image selectors, to study the effect of expertise in the choice of sufficiently representative images for a diagnosis.

\section{IMAGE TRANSMISSION}

Images were sent using the internet multimedia electronic mail, which is based on the MIME (multipurpose internet mail extensions ${ }^{9}$ ) standard system. Briefly, MIME describes a message format for electronic mail which allows the insertion of multiple heterogeneous multimedia components inside a message, which can be transferred by means of the usual email protocols - that is, SMTP (simple mail transfer protocol ${ }^{10}$ ) and POP (post office protocol $\left.{ }^{11}\right)$. The resulting message can be composed of sequences of different media types, even if nested or alternatively displayed. Up to now, such a message format has been partially supported by several mail user agents through the concept of "attachment," which only allows sequences of documents to be appended to a normal message. Although this only forms a subset of the capabilities of MIME, it is sufficient for static telepathology involving transmission of some text data (such as the clinical history) followed by a set of images sampled from the glass slides pertaining to the case.

In order to reduce the storage size needed for images, they can be reduced up to 20 -fold using compression methods such as JPEG. ${ }^{12}$ Compression levels of this degree are achieved by eliminating redundant data and visual information that is unlikely to be useful for image interpretation by the human eye. Although originally intended for standard photography compression, the algorithm used has proved adequate for histological images as well. ${ }^{13}$

\section{TELEPATHOLOGY WORKSTATIONS}

The referring telepathology site was equipped as follows.

- a Powermacintosh 8500 (Apple Macintosh), with embedded framegrabber able to acquire images at $768 \times 576$ pixels, 24 bit colour;

- a Sony 3CCD camera connected to an Olympus Provis AX70 microscope;

- connection to the internet by means of the university network;

- Eudora Light, Macintosh version, as mail user agent;

- standard system software for image acquisition. 
Table 3 Summary of critical cases

\begin{tabular}{|c|c|c|c|c|c|c|c|}
\hline \multirow[b]{2}{*}{ Case } & \multirow[b]{2}{*}{ Selector } & \multirow[b]{2}{*}{ Clinical data } & \multicolumn{2}{|l|}{ Referring site } & \multicolumn{3}{|l|}{ Consultant site } \\
\hline & & & Frozen section diagnosis & Definitive diagnosis & Telepathology diagnosis & Frozen section diagnosis & Definitive diagnosis \\
\hline 1 & $\mathrm{~A}$ & $\mathrm{~F}, 57 \mathrm{y}$, thyroid & Adenoma & Adenoma & Postponed & Adenoma & Adenoma \\
\hline 2 & A & $\mathrm{M}, 77 \mathrm{y}$, rectum & Squamous ca & Squamous ca & $\begin{array}{l}\text { CIS, lack of low } \\
\text { magnification images }\end{array}$ & Squamous ca & Squamous ca \\
\hline 3 & A & $\mathrm{M}, 72 \mathrm{y}$, lung & Infarct & Infarct & $\begin{array}{l}\text { Provisional diagnosis no } \\
\text { neoplasia }\end{array}$ & No neoplasia & Infarct \\
\hline 4 & B & $\mathrm{F}, 76 \mathrm{y}$, breast & Mucinous ca & Mucinous ca & No neoplasia & Postponed & Mucinous ca \\
\hline 5 & $\mathrm{~B}$ & $\mathrm{~F}, 40 \mathrm{y}$, thyroid & Carcinoma & Carcinoma & Neoplasia? Postponed & Carcinoma & Carcinoma \\
\hline 6 & B & $\mathrm{F}, 55 \mathrm{y}$, ovary & Cystadenoma & Cystadenoma & $\begin{array}{l}\text { Cystadenoma. No } \\
\text { images of epithelium }\end{array}$ & Cystadenoma & Cystadenoma \\
\hline 7 & B & $\mathrm{F}, 65 \mathrm{y}$, breast & Postponed & Intraductal ca & Intraductal ca & Neoplasia, postponed & $\begin{array}{l}\text { (Infiltrating?) } \\
\text { papillary ca }\end{array}$ \\
\hline 8 & B & $\mathrm{M}, 63 \mathrm{y}$, diaphragm & Neoplasia & Adenoca & Inflammation & Inflammation & Adenoca \\
\hline 9 & $\mathrm{~B}$ & $\mathrm{~F}, 62 \mathrm{y}$, peritoneum & Adenoca & Adenoca & Postponed & Adenoca & Adenoca \\
\hline 10 & $\mathrm{C}$ & $\mathrm{F}, 52 \mathrm{y}$, ovary & $\begin{array}{l}\text { Serous tumour of } \\
\text { borderline malignancy } \\
\text { and serous adenoca }\end{array}$ & $\begin{array}{l}\text { Serous tumour of } \\
\text { borderline } \\
\text { malignancy }\end{array}$ & $\begin{array}{l}\text { Serous tumour of } \\
\text { borderline malignancy } \\
\text { and serous ca }\end{array}$ & $\begin{array}{l}\text { Serous tumour of } \\
\text { borderline malignancy } \\
\text { and serous adenoca }\end{array}$ & $\begin{array}{l}\text { Serous tumour of } \\
\text { borderline } \\
\text { malignancy }\end{array}$ \\
\hline 11 & $\mathrm{C}$ & $\mathrm{F}, 45 \mathrm{y}$, ovary & Endometriosis & Endometriosis & $\begin{array}{l}\text { Postponed; images } \\
\text { insufficient }\end{array}$ & Endometriosis & Endometriosis \\
\hline
\end{tabular}

A, expert pathologist; Adenoca, adenocarcinoma; B, junior pathologist; C, first year resident; ca, carcinoma; CIS, carcinoma in situ; F, female; M, male; y, years.

The consultant telepathology site was equipped as follows:

- an Olidata P100 computer with Windows 95;

- intermittent internet connection through a $28800 \mathrm{bit} / \mathrm{s}$ modem;

- Eudora Light, Windows version, as mail user agent;

- PaintShop Pro as image visualisation tool.

These different setups also allowed us to verify the interconnection capabilities of two different computers.

CASES

In all, 155 cases were assessed. These were obtained from 142 patients and were submitted to the consultant site as follows: selector A, expert pathologist: 50 cases from 46 patients; selector B, junior pathologist: 54 cases from 50 patients; selector C, first year resident: 51 cases from 46 patients.

Table 1 shows a summary of cases by organ/ system and referring pathologist.

Each pathologist sent a multimedia message to the consultant site composed of a section of text (containing demographic and clinical data) and a series of representative images from frozen section specimens, stained with rapid haematoxylin and eosin (H\&E) or toluidine blue or both.

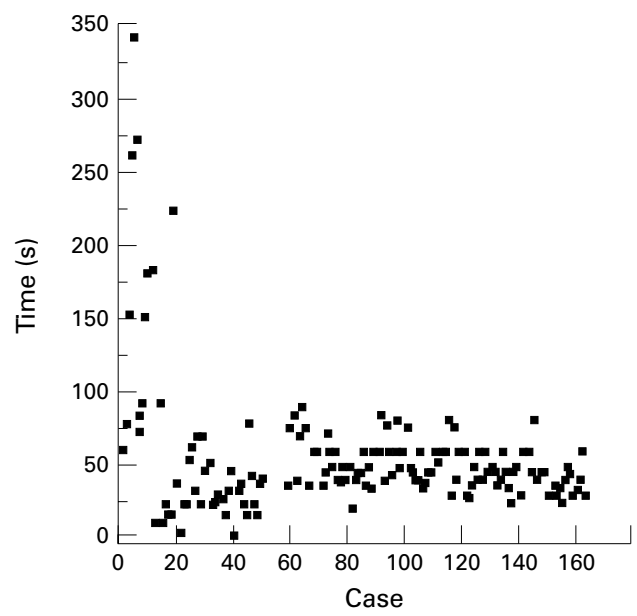

Figure 1 Image acquisition time per case.
STATISTICAL EVALUATION

Five diagnoses were recorded for each case: the local diagnosis on the frozen sections, the gold standard (represented by the local diagnosis on definitive sections); the telepathological diagnosis obtained on the sampled images; the control diagnosis made by the consultant pathologists on the frozen sections, and the control diagnosis made by the consultant pathologists on the definitive sections.

The acquisition and delivery times were recorded, as well as the number of images and overall dimension (in kbytes).

Delivery time comprised three separate times: the time taken to transmit from the referring workstation to the server through the local area network; the time taken to transmit from the Udine server to the Trento server through the internet; and the time taken to transmit from the server to the consultant workstation through the modem connection.

Diagnoses were compared using kappa ( $\kappa)$ statistics; other data are described using medians and quartiles.

\section{Results}

Four of the 155 cases $(2.5 \%)$ were considered inadequate by the remote pathologist and so the diagnosis was postponed in these cases. The telepathology diagnosis agreed with the gold standard in 146 of the 151 remaining cases $(96.7 \%)$.

On average, each case comprised five images (range four to six), with a total dimension of 262 (194 to 330) kbytes. The mean time needed for the acquisition of the images for each case was four minutes (range three to six); the transmission was accomplished in 19 (12 to 33) minutes, for a resulting overall time of 24 (16 to 39 ) minutes. However, maximum transmission time could be up to hours if there were network problems.

Table 2 shows the results obtained broken down by the level of experience of the local pathologists. There were minor differences between the pathologists. The most significant cases in which there was failure to make a diagnosis or postponement are listed in table 3, which gives the five different diagnoses for each 

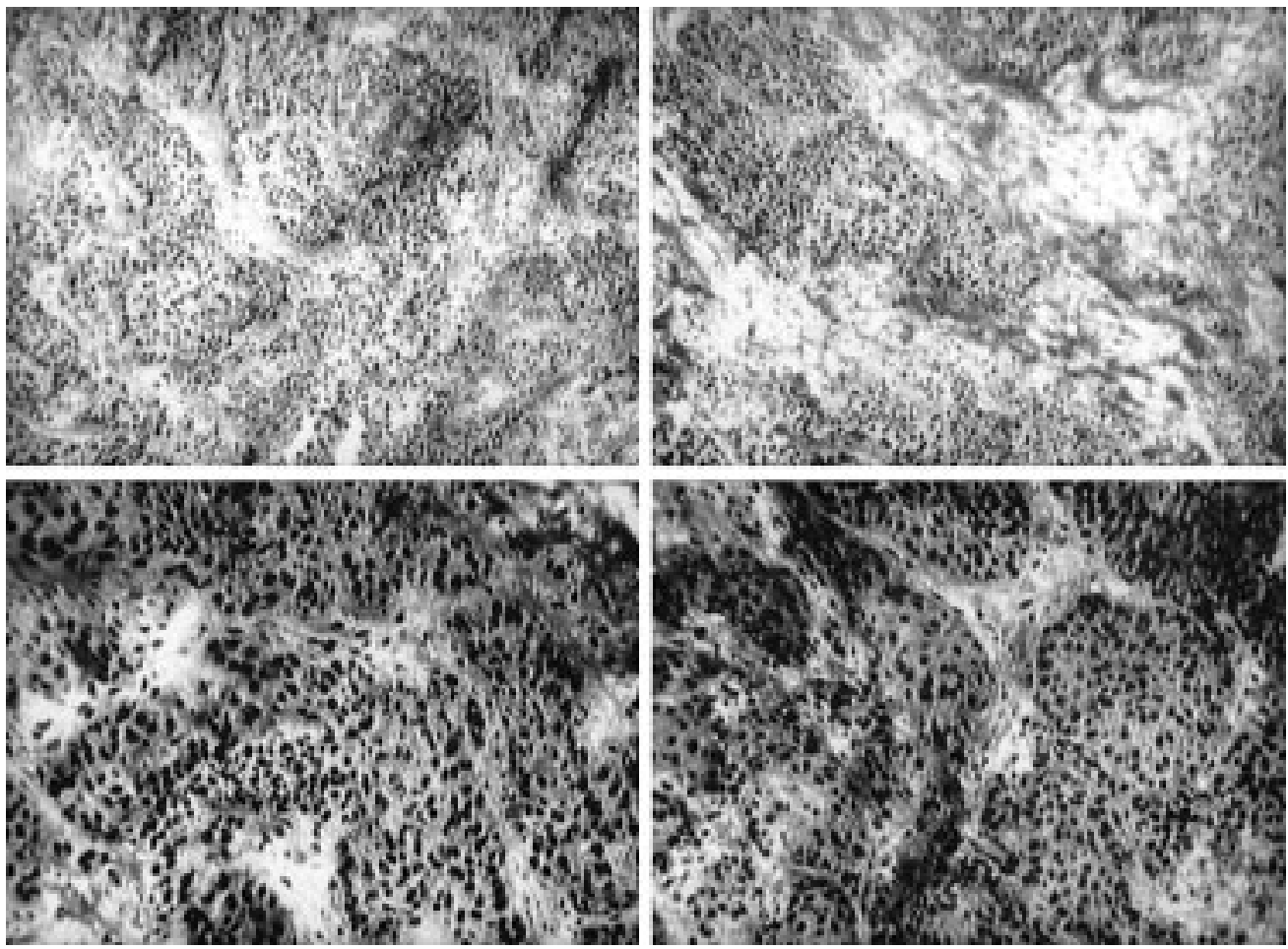

Figure 2 Images required for a representative case (invasive carcinoma of the breast, HEE stain).

case at both sites. In some cases these differences were typical of the problems that are likely to occur when doing static consultations:

- Case 2: images were sampled by the expert pathologist but considered insufficient by the consultants owing to the lack of a low magnification overview;

- Case 4: the telepathology diagnosis was wrong; however, the consultant postponed the diagnosis on the frozen section;

- Case 6: although the telepathology diagnosis was correct, the consultants noted a lack of images;

- Case 7: both the local and consultant diagnoses were deferred, while the telepathology diagnosis was problematic;

- Case 8: in this case the diagnosis of malignancy of the local site was not confirmed by consultants on either telepathological or glass slide diagnosis on frozen section, but they agreed on the definitive diagnosis;

- Case 9: the telepathological diagnosis was postponed, in contrast to that made on the glass slide, thus confirming that the telepathology images were inadequate;

- Case 10: an overestimation of malignancy was made on both the telepathological diagnosis and the frozen section glass slides; this was corrected on the definitive specimen;

- Case 11: a lack of representative images made it impossible for the consultant pathologist to make a diagnosis.

The mean number of images sampled in the failed or postponed cases was the same as in the correctly diagnosed cases.

Dependence of diagnostic performance on image sampling was compared in five main diagnostic classes: negative, non-neoplastic diseases, benign tumours, malignant tumours, and infiltrating tumours. The frozen section and remote diagnoses classified in this way were compared using the $\kappa$ statistic, with similar results for all three selectors (selector A: $\kappa=1$; selector $\mathrm{B}$ : $\kappa=0.97$; selector $\mathrm{C}$ : $\kappa=0.97$, all $\mathrm{p}<0.0001)$.

The time needed for image acquisition appeared to decrease with experience with the method, as can be seen in fig 1; however, this trend did not reach significance.

Figure 2 shows a typical case (breast, invasive carcinoma) represented by a set of four images.

\section{Discussion}

These results indicate that in the routine diagnostic work of a frozen section service, an inexperienced pathologist can sample images sufficiently well to enable a correct remote diagnosis to be made in a reasonably short time. Improved technical quality of the images, obviously preferred by the consultant pathologist, can be achieved with some training in videomicroscopy, though this appears not to be mandatory for the quality of the diagnosis.

Though the median time needed to transmit a single case could be short, the internet cannot be relied on on a regular basis because of unanticipated delays; thus it cannot be used for time dependent tasks such as frozen section telediagnosis. However, the same setup could be adopted for a intranet system, where better performance is achievable.

Our study investigated basic telepathology technique, where an exchange of images occurred without any interaction between the local and the remote pathologist. Despite this lack of interaction, the level of accuracy shown by the method is fairly high, providing a sound 
basis for a frozen section telepathology service where static telepathology is integrated with an online communication module (comprising phone and electronic whiteboard facilitiesthat is, shared cursor and drawing tools) between local and remote pathologists.

When comparing static and dynamic telepathology, two main issues arise: the apparently better performance of a dynamic system versus the lower cost of a static one. As both these techniques remain controversial, ${ }^{46}{ }^{14-17}$ it may be useful to continue assessing them in tandem to compare their applicability, advantages, and limitations. There is renewed interest in low cost telemedicine methods, which is encouraging the development of alternative low cost technologies where possible ${ }^{18-21}$ to allow the use of telemedicine in developing countries. The approach described here can be adopted in other telepathology applications that are currently the subject of research and development-for example, consensus diagnosis, teaching, and quality control-in a more effective way than dynamic approaches, which are too expensive for widespread application.

The technique proposed can be considered inexpensive because it is based on tools widely available in any laboratory; however, as with every telemedicine application, its effect on healthcare costs needs accurate evaluation, though it seems likely that the technique could have a positive impact on costs in comparison with other more expensive systems.

The medicolegal and ethical issues related to telemedicine and telepathology are currently the subject of much interest. ${ }^{22}$ Provided that the telemedicine method is capable of fulfilling the legal requirements, most of these issues are related to the usual medicolegal problems involving devolvement of responsibility during expert consultations. The usual approach is to give the referring pathologist the responsibility of issuing the report, even if it is based on the consultant's opinion. In a comparison between dynamic and static systems, it has been suggested that the consultant pathologist should store the images received as a legal record, in order to protect himself from being sued. However, as pointed out by Sowter and Wells, ${ }^{17}$ the same requirement is involved in every telemedicine transaction, and it is easier to store static images than video. However, the only survey on pathologists' acceptance of telepathology seems to indicate that there is mistrust of still image telepathology ${ }^{23}$ satisfactory image sampling being the major difficulty hindering diagnostic teleconsultations.
In conclusion, there are good reasons to hope that still image telepathology will find an application; thus there should be a commitment to undertake a thorough study of its diagnostic efficacy and cost-effectiveness, as well as the legal issues involved.

This work was partially funded by the Consorzio Universitario del Friuli and by the National Research Council (CNR).

1 Rosen PP. Special report: perils, problems and minimum requirements in shipping pathology slides. Am $\mathcal{F}$ Clin Pathol 1989;91:348-54

2 Abt AB, Abt LG, Olt GJ. The effect of interinstitution anatomic pathology consultation on patient care. Arch Pathol Lab Med 1995;119:514-17.

3 Safrin RE, Bark CJ. Surgical pathology signout. Routine review of every case by a second pathologist. Am f Surg Pathol 1993;17:1190-2.

4 Dervan P, Wootton R. Diagnostic telepathology. Histopathology 1998;32:195-8.

5 Della Mea V, Beltrami CA. Diagnostic telepathology through the internet [letter]. Histopathology 1998;33:485.

6 Weimberg DS, Allaert FA, Dusserre P, et al. Telepathology by means of digital still images: an international validation study. Hum Pathol 1996;27:111-18.

7 Nordrum I. Real-time diagnoses in telepathology. Adv Clin Pathol 1998;2:127-31.

8 Della Mea V, Beltrami CA. Telepathology applications of the internet multimedia electronic mail. Med Inf 1998;23: 237-44.

9 Freed N, Borenstein N. Multipurpose Internet Mail Extensions (MIME) part one: format of internet message bodies. Network Information Center: RFC2045, 1996 (ftp://ftp.isi.edu/innotes/rfc2045.txt)

10 Postel J. Simple mail transfer protocol. Network Information Center: RFC821 - STD10, 1982 (ftp://ftp.isi.edu/in-notes/ std/std10.txt)

11 Myers J, Rose M. Post office protocol-version 3. Network Information Center: RFC1939, 1996 (ftp://ftp.isi.edu/innotes/rfc1939.txt).

12 Wallace GK. The JPEG still picture compression standard. Commun ACM 1991;34:30-44.

13 Foran DJ, Meer PP, Papathomas T, et al. Compression guidelined for diagnosic telepathology. IEEE Trans Inf Technol Biomed 1997;1:55-60.

14 Weinstein RS. Static image telepathology in perspective. Hum Pathol 1996;27:99-101.

15 Raab S, Robinson RA, Snider TE, et al. Telepathologic review: utility, diagnostic accuracy, and interobserver variability on a difficult case consultation service. Mod Path 1997; 10:630-5.

16 Callas PW, Leslie KO, Mattia AR, et al. Diagnostic accuracy of a rural live video telepathology system. Am f Surg Pathol 1997;21:812-19

17 Sowter C, Wells CA. Telepathology: assessment of the implications and applications of telepathology for practical diagnostic pathology. 7 Clin Pathol 1998;51:714-15.

18 Cross M. Telemedicine: choosing the right technology. Health Data Management 1996;4:75-7.

19 Perednia DA. Reinventing telemedicine: store-and-forward applications. Telemed Telehealth Networks 1996;2:15-18, 41.

20 Fredriksen PR, Pettersen S, Pedersen S. Store and forward multimedia in primary healthcare. In: Arvanitis TN, Watson D, eds. Proceedings of the 1st World Congress of the Internet in Medicine, Brighton, UK, November 1997. Brighton: University of Sussex, 1997:58

21 Vazir H, Loane MA, Wootton R. A pilot study of low-cost dynamic telepathology using the public telephone network. Adv Clin Pathol 1998;2:151.

22 Stanberry B. The legal and ethical aspects of telemedicine. London: RSM Press, 1998

23 Mairinger T, Netzer TT, Schoner W, et al. Pathologists' attitudes to implementing telepathology. F Telemed Telecare $1998 ; 4: 41-6$. 